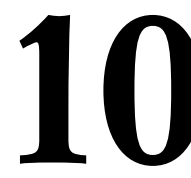

\title{
EL DESEMPEÑO DE LA DIRECCIÓN EN LA UNIVERSIDAD: EL CASO DE DECANOS Y DIRECTORES DE DEPARTAMENTO
}

\author{
(MANAGEMENT PERFORMANCE AT UNIVERSITIES: THE CASE \\ OF DEANS AND DEPARTMENT HEADS)
}

Diego Castro y Marina Tomás

Universitat Autònoma de Barcelona

\section{RESUMEN}

Este artículo presenta los resultados de una investigación cuyo objetivo principal es examinar el desarrollo de la dirección de los órganos unipersonales de gestión académica en la universidad, más concretamente se centra en las particularidades de directores de centro y departamento. Se ha optado por una metodología cualitativa y los datos se obtuvieron a partir de 23 entrevistas y un grupo focal de discusión. Los resultados obtenidos indican que se pueden identificar tres fases en el desempeño de la función directiva de estos cargos. Una etapa inicial, caracterizada por la necesidad de familiarizarse con el cargo, comprender las dinámicas institucionales y en la que se ejerce un liderazgo de baja intensidad. Una segunda etapa, la más extensa en el tiempo, donde la actividad se orienta al desarrollo del proyecto y se caracteriza por una gran dedicación al apoyo y asesoramiento del profesorado. En la tercera etapa aparece la preocupación por asegurar el relevo institucional y por hacer evaluación del impacto de los logros alcanzados.

\section{ABSTRACT}

This article presents the outcomes of a research whose main objective is to examine the professional development of the individual officers of academic management in HE. More concretely, it aims to gain a deeper insight into the particularities of the professional development of deans and head of departments. The data, fundamentally qualitative in nature, come from interviews 
conducted with manager-academics, experts and managers-administrators. The results obtained indicate that three stages can be identified in the way manager-academics carry out their duties. An initial stage is characterised by the need to familiarise themselves with the post, and to understand the dynamics of the institution, during which a low-intensity of leadership is exercised. A second stage, covering the longest period of time, in which all the activity is orientated towards carrying out the project, is characterised by a high level of commitment to providing advice and support to the lecturing staff. The third stage is characterised by the preoccupation with ensuring the institutional hand-over and assessing the achievements attained.

\section{INTRODUCCIÓN}

Presentamos una investigación en la que se relaciona el desarrollo de la función directiva de los/as decanos/as y directores/as de departamento con las dificultades y bondades del modelo directivo actualmente en vigor en la universidad española. La investigación se contextualiza en el actual debate sobre los modelos de gobierno de las universidades públicas (Middlehurst, 2004) y la necesidad generalizada de repensar las estructuras de gobierno de la universidad.

Existe una crisis de confianza de los gobiernos de los estados en relación al actual sistema de gestión de las instituciones de educación superior por lo que las universidades se están viendo sometidas a un proceso de revisión de su modelo de gobierno y rendición de cuentas. Por su parte, Bricall $(2000,418)$ señala que se detectan ciertas tendencias de carácter general que parecen orientarse en una misma dirección desde puntos de partida y desde tradiciones de sistemas universitarios muy diversos:

- Aumento de autonomía institucional correlativa a un aumento del control social sobre las universidades, a través de órganos de gobierno universitario independientes, que incluyen representantes del resto de la sociedad y de la administración pública y de la práctica de la evaluación externa.

- Profesionalización de la gestión interna de las universidades.

- Introducción de estímulos a la innovación en la gestión de estas instituciones universitarias, potenciando la diversificación y la competencia entre ellos.

La tendencia creciente a la autonomía universitaria ha hecho que las universidades hayan asumido muchas de las responsabilidades de gestión que anteriormente tenía la administración educativa. Las universidades en la 
actualidad han demostrado que son capaces de responder adecuadamente a las necesidades de la sociedad utilizando responsablemente los recursos asignados y manteniendo niveles de excelencia en docencia e investigación como principales misiones (Ramsden, 1998). Incrementar la autonomía y el correspondiente rendimiento de cuentas ha dado lugar a muchos cambios que han prescindido de los tradicionales modos de autogobierno protagonizados sólo por académicos (Sporn, 2003). Estos nuevos modelos de gobierno redistribuyen la responsabilidad y el poder en la toma de decisiones entre los diferentes "stakeholders" externos e internos. Existen varios mecanismos de coordinación o control relevantes y nuevas formas de gobierno en el sistema universitario que incluyen:

- Las relaciones entre los gobiernos de los estados y las universidades públicas: se trata de volver a definir la autonomía universitaria y establecer las formas de responsabilidad social de las universidades.

- El gobierno de las universidades: la cuestión fundamental aquí se refiere al modelo interno de gobierno universitario y al equilibrio entre los elementos de un modelo colegial y los del modelo de gestión profesional vinculados al proceso de la toma de decisiones.

- La estructura funcional de las universidades: en la actualidad supone concretar el grado de rigidez o de flexibilidad de las estructuras de cada universidad para hacer frente a los cambios del entorno; lo que implica reflexionar sobre la utilidad de la organización de centros, departamentos, etc.

El modelo de gobierno de la universidad española enfatiza el poder de decisión del profesorado, es el denominado modelo de corporación académica. Bajo este modelo el poder lo ostenta el profesorado permanente a través de representantes o comisiones académicas de las distintas facultades y departamentos, aunque también se han planteado sistemas híbridos (Moore, 1975; Gilmour, 1991; Lee, 1991; Griffith, 1993; Trow,1998; Miller,1999; y Jordan, 2001). La razón esgrimida para justificar la perpetuación de este modelo, además de las de tipo corporativo, es el profundo conocimiento que el profesorado tiene sobre las metas y dinámicas de la institución universitaria (Pfnister, 1970; Williams et al, 1987; Dill and Helm, 1988; Evans, 1999). Evidentemente, este modelo también presenta debilidades que hemos estudiado en esta investigación. Una de las críticas más frecuentes se refiere a que los académicos no están interesados en gobernar la universidad puesto que su motivación se orienta fundamentalmente hacia las tareas relacionadas con la docencia y la investigación. Otro problema que acostumbra a ser identificado es el habitual sistema bicameral que diferencia las dimensiones académica y gerencial (Consejo de Universidades, 1997) y sus correspon- 
dientes disfunciones. Los propios académicos manifiestan falta de formación para el desempeño de la dirección universitaria, e incluso, los rectores muestran falta de formación para dirigir la gestión financiera de una universidad (Smith, 2008). Ahora bien, el reconocimiento de esta deficiente formación no implica forzosamente asumir que el gobierno de las universidades esté viciado. Cuando se han aplicado modelos de gobierno más profesionales y próximos al mercado también han aparecido importantes críticas. En ese sentido Deem (2007), en su investigación sobre directores de departamento, aporta diversas reflexiones al respecto del denominado 'new managerislism' y concluye que este nuevo concepto ha impregnado el quehacer de los profesores que asumen cargos de gestión en la universidad ya que representa un cambio en las relaciones de poder y dominancia. El debate entre el modelo tradicional y otros más profesionales es constante y encuentra su punto débil en la financiación de las universidades. Existe una falta de recursos crónica en las universidades, lo que las inhabilita para adaptarse a las demandas y circunstancias del entorno. Ante las diversas debilidades de los modelos de gestión identificados Trakman (2008) propone un modelo amalgama capaz de incorporar los puntos fuertes de cada uno de los modelos para dar respuesta a las necesidades específicas de cada universidad, este modelo permitiría:

- Realizar investigación básica y crear conocimiento base.

- Incrementar las actividades de ganancias.

- Fondos provenientes del gobierno para propósitos específicos.

- Producir innovaciones que apuntalen el desarrollo económico.

- Asegurar la libertad de los académicos para producir investigación en sus áreas de expertez.

- Construir masa critica en las disciplinas o áreas en que la universidad sea excelente.

- Proveer un contexto donde los estudiantes tengan oportunidades y puedan llegar al máximo de sus posibilidades.

Tras esta panorámica general sobre la universidad como contexto abordaremos, a continuación, la tipología y caracterización de sus órganos de gobierno, especialmente en lo concerniente a los directores de escuela y departamento, objeto de estudio de nuestra investigación. 


\section{2. ÓRGANOS UNIPERSONALES DE GESTIÓN Y GOBIERNO UNIVERSITARIO}

Los órganos de gobierno de las universidades se pueden clasificar en torno a dos variables: composición y ámbito de influencia. Por su composición los órganos de gobierno pueden ser unipersonales o colegiados y, por su ámbito de influencia pueden ser generales ó periféricos (también llamados territoriales). Esquemáticamente se puede representar así:

\begin{tabular}{|c|c|c|c|}
\hline & \multicolumn{2}{|c|}{ Composición } \\
\hline & & Colegiados & Unipersonales \\
\hline \multirow{2}{*}{ 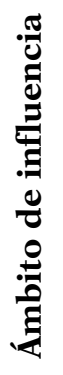 } & $\frac{\sqrt{2}}{\frac{5}{5}}$ & $\begin{array}{l}\text { - Consejo Social } \\
\text { - Consejo Gobierno } \\
\text { - Claustro } \\
\text { - Junta Consultiva }\end{array}$ & $\begin{array}{l}\text { - Rector } \\
\text { - Vicerrectores } \\
\text { - Secretario General } \\
\text { - Gerente }\end{array}$ \\
\hline & $\frac{5}{\frac{5}{2}}$ & $\begin{array}{l}\text { - Junta de Facultad /Escuela } \\
\text { - Consejo de Departamento }\end{array}$ & $\begin{array}{l}\text { - Decano } \\
\text { - Director de Escuela } \\
\text { - Director de Departamento }\end{array}$ \\
\hline
\end{tabular}

Tabla 1. Clasificación de los órganos de gobierno universitario

En esta investigación, como venimos diciendo, se ha profundizado en el estudio de los órganos unipersonales periféricos. Los órganos unipersonales suponen un modelo de liderazgo más próximo al profesorado y a los miembros de la comunidad universitaria.

Ya la LRU (Ley Orgánica 11/1983, de 25 de agosto) atribuía a los decanos y directores de departamento todas las funciones inherentes a la dirección de facultades y departamentos, respectivamente (art. 21) y, establecía que debían ser seleccionados entre el cuerpo de catedráticos o profesores titulares de universidad. Pocas modificaciones incorporó al respecto la Ley 6/2001 de 21 de diciembre, de Universidades, que establecía en sus artículos 24 y 25 el sistema de gobierno de centros y departamentos, respectivamente.

En relación a la dirección departamental Durand (1997) destaca tres aspectos clave:

- La dirección gestiona variables importantes para el desarrollo de la vida de la comunidad académica del área de conocimiento, como por ejemplo: presupuestos, plantillas de personal, formación y evaluación docente. 
- La dirección departamental posee un contacto directo con los administradores, el profesorado y los estudiantes. La dirección del departamento representa un importante agente en la toma de decisiones, partiendo de la idea de que las decisiones se toman más 'abajo' que "arriba", es decir, entre profesores más que entre burócratas. Las decisiones pueden vincularse a aspectos substantivos como la estructura curricular, los programas de formación, la contratación, la promoción del profesorado, los enfoques metodológicos, etc.

En cuanto a la dirección de centros y facultades, Castro (2006) sostiene que actualmente los decanatos asumen dos funciones importantes que son el control general y el seguimiento de los planes de estudio impartidos en sus instalaciones, lo que se concreta en la coordinación de las diferentes variables intervinientes en el desarrollo de las titulaciones.

En este epígrafe también vamos a presentar las características principales en las que ejercen su mandato los órganos unipersonales territoriales; éstas son tres: la elección de tipo representativo, la forma de gestión no profesional y la transitoriedad del cargo.

El primer rasgo es la elección de tipo representativo. El sistema de organización interna, heredado del modelo napoleónico de organización universitaria es básicamente colegial y ha supuesto la concentración del poder institucional en manos del propio profesorado. Esta forma de gestión institucional se identifica con la "burocracia profesional" de Mintzberg (1984), aunque ha incorporado además una ordenación basada en estamentos. Estos estamentos no sólo organizan al profesorado sino también a alumnos y personal de administración y apoyo; aunque el profesorado tampoco es un estamento homogéneo pues los profesores se organizan en función de su rango o nivel académico. Podemos decir que el resultado es una burocracia profesional con rasgos estamentales (Middlehurst, 2004). Este sistema ha hecho que los miembros de la academia elijan a sus directores entre el propio profesorado, generalmente el más senior. En relación a los niveles de autoridad que define Clark (1995) la universidad española ha diferenciado el poder en dos focos: por un lado los académicos de mayor rango (academia) y, por otro, en la administración (gerencia). Estamos de acuerdo con Doland (2003) cuando apunta que la gestión de unidades universitarias debe atender simultáneamente a la administración de la universidad y al cuerpo académico.

El segundo rasgo es la forma de gestión no profesional. La tradición cultural de la representatividad del profesorado implica su presencia mayoritaria en cualquier órgano de toma de decisiones tanto colegial como unipersonal. Los profesores asumen de forma voluntaria y temporal las tareas 
de gobierno institucional de facultades y departamentos conformando un modelo de gestión no profesional (Mora, 2000). Por otra parte, los sistemas internos y externos de evaluación del profesorado han valorado de forma muy superior los méritos de docencia e investigación relegando a un segundo plano las funciones de gestión institucional. Esta tendencia está cambiando de modo que comunidades autónomas y Estado empiezan a valorar la gestión, no sólo en los denominados tramos autonómicos, sino que, por ejemplo es un mérito en el proceso de acreditación para el acceso a las figuras docentes permanentes.

El tercer rasgo es la transitoriedad del cargo. Los profesores una vez elegidos ejercen su mandato durante un periodo de tiempo determinado. La normativa vigente especifica que el mandato de un director de departamento o decano es de tres años, periodo que puede ser prorrogado mediante nueva elección por un segundo mandato. El decano o el director de departamento constituye un jefe temporal que representa y lidera al propio colectivo y actúa siendo consciente de que su poder depende del colectivo que lo nombró (Chaves, 1993). Esta fórmula de gobierno interno es lo que se ha venido denominando en latín un 'Primus inter pares' (el primero entre iguales). La gestión institucional está sometida a periodos de mandato ejercidos por profesorado diferente, esto implica que cada tres o seis años se renuevan los cargos unipersonales y con ellos pueden cambiar las prioridades políticas, las actuaciones y el proyecto de dirección.

\section{CARRERA Y DESARROLLO PROFESIONAL DEL PROFESORADO UNIVERSITARIO}

Las diversas aproximaciones al estudio sobre el desarrollo profesional del profesorado universitario aconseja distinguir los conceptos de carrera académica y desarrollo académico, dos conceptos que a pesar de estar muy vinculados presentan diferencias importantes de matiz. La carrera académica supone la representación estructurada en fases o estadios que organizan una profesión y su dimensión es de naturaleza estructural. El desarrollo académico es el proceso de carácter dinámico que permite un aprendizaje permanente para obtener mayores logros y beneficios en el puesto de trabajo, por tanto responde a una perspectiva más fluctuante e interpretativa (Zabalza, 2002, 135).

En España se carece de carrera académica diseñada convenientemente como tal (Bricall, 2000) a pesar de que las indicaciones de los diferentes informes nacionales desarrollados en Europa, como el de Dearing (1997) en Inglaterra o el de Bricall (2000) en España, defendían que la carrera académica debe orientarse hacia la expectativa natural de promoción profesional. 
En la práctica, el diseño de una carrera académica tiene que estar relacionado con la planificación de los recursos humanos de las universidades, concretamente con la previsión de flujos de entradas y salidas del sistema (Nogueira, 2004).

Huberman y otros (2000) plantean un gran cambio en el enfoque del estudio del desarrollo profesional en la medida que no lo supeditan a la edad cronológica o los años de experiencia profesional. Su taxonomía describe las fases de desarrollo teniendo en consideración aspectos personales de cada sujeto, como el carácter, los rasgos de personalidad, etc. Este enfoque abre la puerta a un planteamiento más global en el que interaccionan todas las variables y dimensiones de la vida del sujeto en todas sus facetas: los ciclos de vida profesional (Oja, 1989; Samper 1992; Fernández Cruz, 2006; etc.). De esa manera entendemos que el desarrollo profesional del docente es una amalgama de aprendizajes acumulados, resultado de la reconstrucción desde la experiencia del pasado y que continúa en el presente.

Kugel (1993) describe una propuesta de 5 etapas sobre el desarrollo del profesor universitario como docente y explora lo que ocurre durante cada etapa. Las tres primeras etapas (Yo, Materia y Estudiante receptor) se centran en la preocupación del docente por la enseñanza del contenido académico y, en las dos restantes, aparece (Estudiante activo y Estudiante independiente) un interés creciente por el proceso de aprendizaje de sus alumnos.

Robertson (1999) propone una clasificación dividida en dos fases: egocentrismo y aliocentrismo. La fase de egocentrismo entronca con toda la perspectiva de las fases iniciales en la carrera docente y por tanto en la centralidad del profesor (sus ideales, tendencias, intereses, miedos, etc.), en tanto que la fase de aliocentrismo supone una superación de la primera y se caracteriza por un interés creciente en el estudiante (cómo aprende, que le interesa, cómo lo hace, etc.). Pero la novedad se la confiere a la taxonomía la dimensión sistémica de rasgos más trascendentales y éticos en los que los protagonistas del proceso educativo (alumnos y profesores) participan y comprenden una experiencia genuinamente humana como es el proceso de formación. Bajo esa óptica las posibilidades y limitaciones del contexto así como una forma de relación horizontal entre los actores adquieren una importancia altamente significativa.

\section{FASES EN EL DESARROLLO DEL PUESTO DE DIRECCIÓN}

El interés por comprender el desarrollo de los cargos de dirección responde tanto a cuestiones de orden institucional como de tipo personal. 
Institucionalmente estos puestos representan los niveles estratégicos de la organización y desarrollan un rol fundamental en centros y departamentos. En cuanto al desarrollo de las personas y su carrera profesional brinda la posibilidad de mejorar la coherencia y articulación entre las funciones asignadas, así como, facilitar la transición entre las diversas fases.

De la misma manera que las organizaciones y las carreras profesionales presentan unos estadios evolutivos propios, el desempeño de los cargos de gestión universitaria no escapa a tal evolución (Turnbull \& Edwards, 2005). El desempeño de un cargo institucional presenta una serie de fases evolutivas caracterizadas por una lógica artificial que resalta el común denominador de las distintas etapas en la evolución de los cargos. Este tipo de taxonomías facilitan la ordenación de la realidad aunque implican su reducción y simplificación. Las etapas en el desarrollo del puesto no suponen la mera superposición de fases y momentos, ya que los rasgos individuales, organizacionales y sociales inciden de forma decisiva en el desarrollo cada persona en el puesto que ocupa (Akerlind, 2008). Las distintas taxonomías sobre el desarrollo de los puestos directivos advierten: 1) La evolución de las etapas no representan un proceso lineal ya que las fases identificadas pueden alterarse o repetirse en función de las influencias de cada persona, los grupos o la cultura organizativa. 2) El desarrollo no es homogéneo y cada persona puede desarrollarse de una manera específica, provocando la pluralidad de situaciones y la complejidad característica de la realidad profesional de cada momento. 3) La caracterización de fases posibilita una perspectiva proyectiva lo que permite su óptima planificación, la previsión de conflictos y su intervención efectiva. 4) Representa un modelo cíclico en la medida que cada incorporación a una nueva responsabilidad o cargo reproduce, en mayor o menor medida, las fases de desarrollo en el puesto.

Robbins (1987) identifica cinco etapas en relación a la actuación de las personas en el desarrollo de puestos o cargos específicos: integración, socialización, mantenimiento, negociación y recuerdo:

- Integración, se produce en el momento de entrada y llegada al nuevo escenario profesional. Es fundamental la integración en la dinámica institucional, y en el sistema de relaciones del puesto: contactos, personas, grupos, presiones que recibe, etc.

- Socialización, implica la aceptación no sólo explícita sino implícita del cargo y de sus condiciones de ejecución. Es importante destacar que durante este periodo se producen procesos de asimilación y acomodación, además se integran las pautas culturales dominantes como propias a la vez que el sujeto proyecta sus formas personales de actuar. 
- Mantenimiento, durante esta etapa se consolida la integración definitiva del sujeto y se amplían sus posibilidades de participación e implicación. El sujeto ya está plenamente consolidado y puede desarrollarse como profesional, los espacios para la innovación y el cambio son característicos de esta fase ya que hay mayor seguridad y consolidación de los aspectos técnicos y relacionales. Máximo dominio y seguridad en las ejecuciones previstas.

- Negociación, la fase de aprendizaje e innovación se paraliza y como consecuencia se genera un primer estancamiento por el cual el sujeto incorpora la negociación para ampliar funciones o nuevas competencias. Si las negociaciones surten efecto se puede producir una redefinición del puesto que puede darle un enfoque novedoso, aunque por poco tiempo ya que el cambio debe producirse a mayor escala. Una vez agotada esta segunda parte del puesto se debate entre un mantenimiento anodino o una nueva promoción o cambio.

- Recuerdo, tras la fase de negociación se inicia una etapa profesional por la que se inicia el ciclo de nuevo. En todo caso la experiencia acumulada y las relaciones entre el sujeto y su puesto han generado ciertas sinergias que pueden consolidarse, e incluso institucionalizarse. Se genera un elemento de reminiscencia, recuerdos y vínculos que pasan a participar tanto del currículo del sujeto como del de la propia institución.

\section{METODOLOGÍA DE LA INVESTIGACIÓN}

La metodología utilizada ha sido fundamentalmente cualitativa y se ha concretado en la aplicación de dos instrumentos: la entrevista y el grupo focal de discusión. La entrevista en profundidad ha servido para obtener los datos y se ha diseñado de forma semiestructurada para focalizar los tópicos fundamentales de la investigación. El grupo de discusión tenía como finalidad disponer de medidas de fiabilidad y validez estructuradas y poder profundizar en las informaciones y sus relaciones de significado emergente para asegurar mayor rigor en el proceso de reconstrucción de los datos.

En la entrevista participaron 23 informantes de las 4 universidades públicas del área metropolitana de Barcelona (Universitat Autònoma de Barcelona, Universitat Politècnica de Catalunya, Universitat Pompeu Fabra i Universitat de Barcelona). Se ha utilizado la triangulación de informantes, siendo sus perfiles: 1) académicos que ejercen funciones de dirección en departamentos o facultades; 2) expertos analistas del gobierno y gestión de la universidad y; 3) personal de administración y servicio vinculado a la direc- 
ción de centros y departamentos. La muestra final se estableció utilizando criterios no probabilísticos basados en la representatividad de los informantes y utilizando el modelo de muestreo teórico (Flick, 2004). Las cuestiones que abordaba la entrevista indagaban sobre la importancia, funciones y trascendencia del cargo; condiciones en la que se ejerce la dirección universitaria; forma de acceso y desarrollo; problemáticas y dificultades en la evolución del cargo, etc. La entrevista nos permitió la obtención de datos que fueron administrados y sistematizados con la ayuda de las aplicaciones del programa Aquad versión 6. Tras el proceso de análisis se elaboró un primer informe preliminar que permitió la focalización del debate del segundo instrumento. El grupo focal se orientó a la discusión del informe previamente remitido y se constituyó con la participación de nueve informantes expertos en la dirección y gestión de instituciones de educación superior. Para el análisis intensivo de los datos recogidos de ambos instrumentos se recurrió a los planteamientos metodológicos de la Teoría Fundamentada de datos (Tesch, 1990). El sistema de análisis fue construido de forma complementaria entre planteamientos deductivos (previos al propio trabajo de campo como los objetivos de investigación, los tópicos abordados en el marco teórico, etc.) e inductivos (datos obtenidos de las entrevistas y grupos de discusión y las relaciones conceptuales que se establecen entre los tópicos de significado, etc.).

\section{ANÁLISIS DE LOS RESULTADOS}

El desempeño de cargos de gestión unipersonal en la universidad no representa una realidad estática, sino que se caracteriza por un desarrollo más o menos estructurado. En esta investigación hemos podido identificar algunas de esas fases de evolución y sus características principales.

a) Etapa Inicial. Supone la contextualización del profesor en el nuevo rol directivo y se produce a través de tres procesos: uno, la necesidad de conocer el contexto macro y micro institucional; dos, comprendiendo y analizando las dinámicas grupales y personales y; tres, interiorizando el trabajo rutinario y los procesos burocráticos.

Hay un primer periodo en el que tienes que hacerte con todo el volumen de trabajo, de encargos y de responsabilidades que tienes. En el momento de coger la responsabilidad, es un periodo que es bastante largo en relación al período total, yo calculo que es un curso escolar, porque todo absolutamente es nuevo, desde asistir a las reuniones del rectorado a dirigir la secretaría y como en la universidad nos movemos por cursos, todo lo que ocurre el primer año es nuevo y como se puede decir que no lo sabes, lo has de afrontar.

(Susana, decana de facultad) 
Del estudio detallado de los datos emergen hasta 3 características definitorias de la etapa inicial: alta motivación, prudencia en la toma de decisiones y un ejercicio de poder que se podría denominar de 'baja intensidad'. Existe coincidencia en las entrevistas al definir esta etapa como una de las más interesantes por la alta motivación y por las altas expectativas. El afán por ejecutar actuaciones y poner en práctica ideas y políticas coincide con la actuación cautelosa y prudente. La falta de experiencia y el desconocimiento en algunos temas aconseja decidir sólo sobre cuestiones urgentes e inaplazables, de la misma manera, se acostumbra a ser bastante continuista con las directrices anteriores y no se suelen desarrollar actuaciones excesivamente rupturistas.

La duración cronológica de esta etapa puede asociarse a la conclusión del primer ciclo académico-administrativo completo, pues supondría el ejercicio completo de todas las tareas y funciones propias del cargo.

En la primera fase tú ya entras en la dirección con unas ideas de lo que quieres hacer, probablemente combinándolas con una cierta prudencia de conocer un poco como es el terreno antes de ver la viabilidad de cuando tú preparas el programa electoral no conoces el terreno y a lo mejor puedes estar diciendo sandeces. Por lo tanto se podría decir que la primera fase se caracteriza por una cierta expectativa elevada de cosas que quieres hacer más una prudencia de observar primero como está la situación.

(Juan, director de departamento)

Los directivos manifiestan que se enfrentan a dos dificultades que les provocan cierta inseguridad durante esta etapa inicial. La primera gira entorno a la conducción de un determinado tipo de reuniones, especialmente las muy numerosas o las que cuya temática se prevé conflictiva. En segundo lugar, les genera cierta inseguridad cometer errores por desconocimiento de la normativa vigente en cada temática. Estos temores hacen que los directivos inicien procesos de negociación o consenso previos a las reuniones con determinado profesorado o con grupos de interés para evitar una situación de conflicto o su desgaste como líder.

Por tanto es un primer año en que es difícil porque no se está preparado para afrontarlo. Después hay unas dinámicas, unos procedimientos que están instalados en el personal de administración y servicios, y que son del día a día, y que te sorprenden y hasta que no las tienes interiorizadas y puedes establecer estrategias de actuación, ya te encuentras en el segundo año en el cual ya llevas la dinámica, que tú como dirección, quieres implementar.

(Carmen, directora de escuela universitaria) 
Aparecen como funciones principales en esta etapa el conocimiento profundo de las distintas unidades organizativas (grupos, personas, comisiones), el diagnóstico de los equipos para mejorar su cohesión, la difusión del proyecto de dirección y las reuniones de trabajo con los colaboradores próximos para apuntar las acciones prioritarias. Otras responsabilidades de la etapa inicial son la observación constante y el diálogo abierto con todos los miembros de la organización. Se identifica que es un periodo de aprendizaje rápido pues hay que actualizarse en todos los asuntos.

La verdad es que es ir observando un poco a ver que pasa. No tomar por supuesto ninguna decisión. Lo primero que hice fue reunirme con todos los departamentos y escucharlos y acercarme a ellos y no esperar a que ellos se acercaran a mí porque había un poco de distanciamiento.

(Susana, decana de facultad)

b) Etapa de Desarrollo. Es un periodo caracterizado por el intensivo desempeño de las funciones que tiene atribuidas el cargo. La legislación vigente y los estatutos universitarios asignan unas funciones diferentes a ambos cargos unipersonales (decano y director de departamento). Por ello los entrevistados han diferenciado entre el desarrollo del cargo en la dirección de un centro o facultad de la dirección de un departamento.

Buena parte son cuestiones derivadas de la representación, desde profesores visitantes a contactos con empresas, contactos de los estudios fuera de la casa, captación de nuevo estudiantado. Luego por otra parte está todo lo que tiene que ver con el desarrollo interno del programa y era eso a lo que me refería con la cuestión de la integración en el espacio europeo, etc. También tengo la obligación de acudir a reuniones con rectorado. Tengo una agenda comprometida por visitas, por reuniones y por el propio trabajo.

(Ignacio, decano de facultad)

Las responsabilidades directivas en esta etapa se pueden organizar en dos grandes ámbitos. El primero afecta a las funciones de tipo institucional: reuniones y comisiones de representación, la intervención en la resolución de conflictos, la comunicación interna y externa, el cumplimiento de los procesos burocráticos y los procesos de negociación (especialmente en la demanda de recursos). El segundo engloba la gestión de personal docente aunque también deben intervenir en situaciones vinculadas al personal de administración y servicios. Se define esta etapa como un periodo de máxima actividad y muy estresante en el que el académico-manager debe atender a todas las funciones asignadas. 
Me abruma muchas veces la sensación de responsabilidad y no poder dejar pasar ningún tema, aunque no puedo dedicarme en detalle a ninguno de ellos, porque para eso está el equipo directivo, y porque, ya no doy más de sí. Y entonces uno tiene que dominar bien el arte de la delegación, de la confianza en los demás y de aceptar un trabajo que sabes que, si lo hubieras hecho tú a lo mejor pudiera haber estado un poquito mejor, pero yo ya no puedo hacer nada más, aunque ese tipo de cosas requieren unos cambios mentales y de perspectiva que no son siempre fáciles.

(Agnes, decana de facultad)

El liderazgo durante esta etapa se orienta a potenciar nuevos proyectos, la posibilidad de ejercer un mayor rol de control sobre personas y recursos y, también, por un aumento de la delegación en determinados asuntos. Los directores de centro y departamento que deben aplicar medidas sancionadoras durante esta etapa confiesan hacerlo con mayor tranquilidad y decisión aunque consideran que sólo se dan en circunstancias excepcionales. Existe una mayor iniciativa en la capacidad de actuación y se observa una mayor confianza en las posibilidades personales y en las del propio equipo. La experiencia y el aprendizaje permiten proponer iniciativas innovadoras que substituyen modelos y prácticas aplicadas en anteriores mandatos.

c) Etapa Final. Denominada también de salida es seguramente la que ocupa un periodo más breve de las tres que hemos identificado. Se refiere al periodo de tiempo que va desde que el directivo decide no presentarse a la reelección hasta el momento en que se convoca la reunión para la nueva elección. En la etapa final se identifican tres secuencias diferenciadas. La primera es la que denominamos de preparación para la salida. Implica el cierre de los proyectos en curso y la preparación de la memoria final. Las iniciativas y las nuevas propuestas se paralizan ante la inminencia del relevo. La segunda es la que se vincula al procedimiento legal del relevo. La preparación de nuevas candidaturas, el proceso administrativo establecido por los estatutos universitarios y las elecciones son otra secuencia importante. Las tareas de relevo son consideradas de máxima importancia por el profesorado entrevistado ya que las consideran un factor que garantiza un traspaso exitoso del poder y garantía democrática. La tercera secuencia es la denominada propiamente de salida. Supone el final del mandato y presenta tres rasgos característicos. El primero de tipo formal implica el relevo de la dirección. El segundo de tipo funcional supone el traspaso de los temas y asuntos pendientes. El tercero de naturaleza personal conlleva el retorno del profesor a sus labores como docente e investigador. El directivo se convierte de nuevo en un miembro más del departamento o escuela. Hemos podido constatar en las entrevistas la poca tendencia que se da para desempeñar va- 
rios mandatos consecutivos. Los cargos unipersonales consideran que el cargo requiere un alto sacrificio personal y profesional. La reelección en varios mandatos acabaría afectando negativamente a sus carreras.

En tres años, lo que quieres hacer puedes hacerlo. Si no lo haces en tres años, no lo harás en seis, y seis años son muchos años. En principio, yo no me veo a mí misma volviéndome a presentar para decana, en absoluto.

(Isabel, decana de facultad)

En cualquier caso los motivos por los que un decano o jefe de departamento se plantearía volver a presentarse a la dirección son: por no haber podido acabar con los proyectos iniciados, un segundo motivo sería tener asegurado un cierto apoyo por parte de la institución para la continuidad y, finalmente, que no hayan aparecido otras posibles candidaturas para ocupar el cargo. Estos resultados corroboran el diagnóstico en el que se presentaba la gestión como una función compleja que requiere mucho tiempo y que supone paralizar el resto de funciones que constituyen el perfil profesional del profesorado universitario.

En el relevo de la dirección universitaria se manifiesta como un valor implícito la experiencia demostrada del candidato en funciones institucionales anteriores de igual o menor categoría. Casi no hemos encontrado cargos de decano o director de departamento que inicien su actividad directiva sin experiencia en algún tipo de responsabilidad anterior. Este 'modus operandi' es ampliamente defendido por la comunidad universitaria pues asegura modelos no rupturistas y una continuidad de los principios institucionales. Además lleva implícito un cierto modelo de formación para la gestión.

Tras la etapa final se cuestionan algunos aspectos del actual modelo vigente de cargos de gestión unipersonal y cómo éste influye en sus etapas de desarrollo. La mayoría de los Estatutos de las universidades públicas españolas conceden a los cargos de gestión unipersonal tras el final del mandato un periodo de exención de docencia que comprende aproximadamente un curso académico completo. El periodo sabático debe invertirse fundamentalmente en la actualización científica, artística o técnica del profesorado. Las personas que han participado en esta investigación no consideran que tras el mandato el retorno en exclusiva a las funciones de docencia e investigación provoque disfunciones en el funcionamiento de los departamentos y facultades. Los argumentos que se dan para explicar la naturalidad del retorno a las tareas de docencia e investigación son de tres tipos diferentes. El primero es que se retoman en exclusiva las tareas docentes e investigadoras altamente valoradas en la actualidad. 
Yo creo que eso es muy sano tanto para uno mismo como para la institución en general porque entonces hay mucha gente que ha pasado por otros puestos y conoce el sistema y como funciona y comprende mejor otras variables.

(Miguel, director de departamento)

Otro razonamiento que aparece en el análisis de las entrevistas es en relación a la evaluación del desempeño profesional. En la evaluación externa del profesorado priman los méritos de investigación y difusión del conocimiento siendo la gestión un mérito escasamente valorado. El profesorado entrevistado considera imposible compaginar el cargo de gestión con el resto de funciones, especialmente las de investigación. Por ello, la ponderación que en la actualidad se realiza de la actividad investigadora frente a cualquier otra se vive de manera preocupante por el profesorado dedicado a la gestión. Entre este se generaliza la queja sobre el actual sistema que no valora suficientemente la función de gestión. Las personas entrevistadas consideran que en el momento de la evaluación parten de una situación desfavorable pues no pueden aportar evidencias de investigación. Se demanda un modelo que equipare las funciones de docencia, investigación y gestión y que permita una evaluación equitativa de los diferentes méritos aportados. De las entrevistas se desprende la necesidad de proponer un sistema de evaluación integrado por las tres funciones del profesorado durante un periodo concreto. Se trata de comprobar si la actividad que ha realizado el profesorado durante ese periodo es satisfactoria o no sea cual sea la función: docencia, investigación o gestión. De esta manera nos aseguramos que las tres funciones se valoran de forma equitativa.

La gestión es una parte de nuestro trabajo como profesores y debe ser evaluada con pertinencia. No se puede hacer todo bien y todo a la vez. El sistema de ahora es una trampa, hacer gestión no está reconocido en un sistema en el que sólo se valora la investigación. Si sigue así nadie querrá hacerlo. Si te dedicas a la gestión sólo te deben evaluar por desempeño como gestor, no tiene sentido que te castiguen por no hacer investigación. En cada periodo de tu carrera te puedes dedicar a una sola actividad en exclusiva y sólo de esa actividad debes evaluarte. Luego cuando acabes un periodo puedes desarrollar otra función y hacerlo intensamente.

(Mercedes, directora de departamento)

Entre otros motivos la situación de desventaja de la función directiva ante las funciones de docencia e investigación ha provocado que muchos de los procesos para la provisión de nuevos directivos queden sin candidatos. Esta situación es identificada como un serio problema del sistema de gestión vigente en España, por lo que se demandan medidas urgentes para solucionarlo. 


\section{CONCLUSIONES}

Las etapas que permiten describir el desarrollo de los cargos unipersonales de gestión de centros y departamentos coinciden con las teorías existentes en el ámbito de la gestión de recursos humanos. Esta coincidencia abre una nueva perspectiva en la gestión e intervención sobre los directivos a partir de experiencias vigentes y modelos demostrados eficaces en otros contextos no universitarios. Coincidimos con Akerlind (2008) al comprobar como el desarrollo de las etapas permite dibujar perfiles, más o menos generalizables, capaces de caracterizar las funciones, características y limitaciones en cada etapa de ese desarrollo.

Las conclusiones apuntan que las fases que definen la evolución en el desempeño de la dirección de los órganos unipersonales son: 1) etapa inicial, 2) etapa de desarrollo y, 3) etapa de salida.

La etapa inicial del cargo podemos denominarla de contextualización y se asimila a las fases denominadas por Robbins (1987) de integración y socialización. Se produce a través de tres procesos básicos: por un lado la necesidad de conocer el contexto macro y micro institucional; segundo, comprendiendo las dinámicas relacionales de grupos y personas y; finalmente, interiorizando determinados procesos y formas de trabajo más o menos estandarizadas. En esta etapa se constata una alta motivación hacia el cargo. Las actuaciones se desarrollan de forma un tanto continuista con planteamientos propios de mandatos precedentes y se manifiestan ciertos miedos ante determinadas situaciones de conflicto. En relación a las funciones que son consideradas básicas en esta primera etapa del cargo podemos destacar, por un lado, la convocatoria de reuniones y comisiones, tanto para presentar la línea de trabajo como para conocer el estado de cada unidad organizativa (grupos, personas, etc.), y en segundo lugar, el trabajo interno entre todo el equipo adscrito al proyecto (subdirectores, secretarios, vicedecanos, etc.) para fomentar la cohesión y la unidad grupal.

Se ha evidenciado un desconocimiento de la labor y funciones específicas del cargo de dirección por parte del profesorado antes de tomar posesión del cargo. Ese desconocimiento le lleva a conocer durante su mandato algunos de los rasgos que implica la labor directiva en la universidad. Primero la complejidad del cargo y las responsabilidades que conlleva, tomar conciencia de que se está prestando un servicio a la colectividad debiéndose superar ciertas atribuciones previas desenfocadas del cargo, identificar los sistemas de control que delimitan la capacidad de poder real, etc.

La etapa de desarrollo implica mucha actividad y hacer frente a las muy diversas y complejas funciones vinculadas al cargo lo que obliga al 
profesor a dedicarse de forma exclusiva a la dirección. Consideramos que de las tres etapas identificadas es la más extensa y posee un marcado carácter funcional. Esta segunda etapa comprende dos grandes ámbitos de responsabilidad: el primero afecta a las funciones de tipo institucional como convocar juntas, presidir comisiones de representación y mantener los procesos y normativas de carácter burocrático. El segundo hace referencia a la gestión del personal especialmente en lo que se refiere al profesorado. Se destaca especialmente la intervención y resolución de conflictos entre personas y grupos, la gestión de la comunicación, etc. Desde el punto de vista del ejercicio del liderazgo en esta etapa aparece la posibilidad de impulsar nuevas ideas y políticas. Además de la necesidad de ejercer un rol de mayor control.

La etapa de salida o final es la más breve de las que configuran la taxonomía que presentamos si bien, es la que más cuesta acotar temporalmente según los datos obtenidos. En ella se pueden identificar hasta tres secuencias diferentes. Una primera supone la preparación para la salida, en la que empiezan a cerrarse temas o etapas de los proyectos más complejos. Las iniciativas y los nuevos retos de actuación parece que se paralizan ante la inminencia del relevo. En algunos casos se hace referencia al feed back que se establece entre la dirección y el resto de la unidad institucional sobre los logros y actuaciones realizadas, y otros aspectos destacados del mandato. La segunda secuencia es la que se vincula al procedimiento establecido para el relevo institucional. La preparación de nuevas candidaturas, el proceso electoral y las elecciones son otra parte importante que caracteriza a esta etapa. La tercera secuencia es la denominada propiamente como salida, es el final del mandato e implica el relevo de la dirección desde la perspectiva formal y, el traspaso de los temas y asuntos pendientes desde la perspectiva funcional. Tras ese momento la situación profesional para el académico que ha ejercido el cargo cambia substancialmente al tener que retornar en exclusiva a las funciones de docencia e investigación, perspectiva personal. Se podría entender que tras el paso de los académicos por puestos de dirección su perspectiva institucional, e incluso, su sentimiento de pertenencia o su implicación son mayores.

Se ha podido comprobar como cada una de las fases que hemos enumerado pone el énfasis en un aspecto concreto: en la etapa inicial priman los aspectos más personales -el yo del directivo- (alta motivación, necesidad de contextualización, temor al fracaso, etc.). En la etapa de desarrollo prima la perspectiva funcional. Finalmente, en la etapa de salida destaca el compromiso con la institución.

Entre nuestra investigación y los estudios de Kugel (1993), Robertson (1999) y Feixas (2001), sobre las etapas evolutivas del profesor universitario como docente, hemos podido constatar importantes similitudes. En la pri- 
mera etapa se manifiesta una preocupación subjetiva por el propio desempeño; en la segunda se produce una clara preocupación por la eficacia de los logros alcanzados en las funciones propias del cargo y finalmente, en la tercera etapa se constata una clara preocupación por la dimensión institucional.

Tras los años de desempeño de la función directiva se regresa a la vida académica basada en la docencia y la investigación lo cual no parece generar dificultades. Son muy pocos los casos en los que volver a la actividad académica habitual puede acarrear disfunciones y cuando esa circunstancia se da obedece a un sentimiento de pérdida de estatus o a características y rasgos de la personalidad del profesorado. El sistema vigente es, por lo general, especialmente valorado por tres motivos: primero porque permite retomar en exclusiva las tareas docentes e investigadoras, altamente valoradas y prestigiadas (Deem, 2005); segundo porque la alternancia de candidatos demuestra que el sistema representativo y estamental de la universidad es muy sano y realmente funciona y, tercero, porque siempre existe la posibilidad de colaborar y participar con los equipos directivos posteriores.

No hemos obtenido evidencias suficientes para cuestionar el modelo directivo existente aunque sí se han manifestado criticas veladas y algunas demandas de cambio lo que abre nuevas líneas de investigación en esta perspectiva. 


\section{REFERENCIAS BIBLIOGRÁFICAS}

Akerlind, G. (2008). Growing and developing as a university researcher. Higher Education, 55. 241-254.

Bricall, J. M. (2000). Informe Universidad 2000. Madrid: CRUE.

Castro, D. (2006). Los órganos unipersonales de gestión académica en la Universidad. Tesis doctoral. Departamento de Pedagogía Aplicada: UAB.

Chaves, J. R. (1993). Organización y gestión de las universidades públicas. Barcelona: PPU.

Clark, B. (1995). Leadership and innovation in Universities. From Theory to Practice. Tertiary Education and Management, 1(1), 7-11.

Consejo de Universidades (1997). Órganos de gobierno de la universidad. Madrid: Consejo de Universidades.

Dearing, R. (1997). Higher education in the learning society. London: Bof.

Dearlove, J. (1997). The Academic Labour Process: from Collegiality and Professionalism to Managerialism and Proletarianisation? Higher Education Review, 30 (1), 56-76.

Deem, R. (2006). Changing Research Perspectives on the management of Higher education: Can research permeate the activities of manager-academics? Higher Education Quarterly, 60 (3), 203-228.

Deem, R. \& Brehony, K. (2005). Management as ideology: the case of "new managerialism" in higher education. Oxford Review of Education, 31 (2), 217-235.

Dewey, J. (1966). Democracy and Education. New York: The Free Press

Dill, D. \& Helm, K. (1988). Faculty Participation in Strategic Policy Making.
Higher Education: Handbook of Theory and Research, 4, 320-355.

Doland, S. L. (1999). La gestión de los recursos humanos. Madrid: McGrawHill.

Durand, J. C. (1997). Dirección y liderazgo del departamento académico en la universidad. Navarra: EUNSA.

EURYDICE (2008). Higher Education Governance in Europe. Policies, structures, funding and academic staff. Bruselas: European Commission.

Evans, J. P. (1999). Benefits and Barriers to Shared Authority. M. T. Miller (ed.), Responsive Academic Decision-Making: Involving Faculty in Higher Education Governance. Stillwater: New Forum Press.

Feixas, M. (2002). El desenvolupament professional del professor universitari com a docent. Tesis doctoral. Departamento de Pedagogía Aplicada, UAB.

Fernández, M. (2006). Desarrollo Professional docente. Granada: Grupo Editorial.

Flick, U. (2004). Introducción a la investigación cualitativa. Madrid: Morata.

Gilmour, J. E. (1991a). Your Faculty Senate: More Effective Than You Think? Academe, 77 (5), 16-18.

Gilmour, J. E. Jr. (1991b). Participative Governance Bodies in Higher Education: Report of a National Study. New Direction for Higher Education, 19 (3), 29-30.

Griffith, R. (1993). Budget Cuts and Shared Governance. Academe, 79 (6), 15-17.

Hall, M \& Syrnes, A. (2005). South African higher education in the first 
decade of democracy: from cooperative governance to conditional autonomy. Studies in Higher Education, 30 (2), 199-212.

Hancock, T. (2007). The business of universities and the role of department chair. International journal of educational management. 21 (4), 306314.

Huberman, M.; Thompson, C. \& Weiland, S. (2000). Perspectivas de la carrera del profesor, en Biddle, B. J. y otros La enseñanza y los profesores $I$. La profesión de enseñar. Barcelona: Paidós.

Jordan, R. (2001). The Faculty Senate Minuet. Trusteeship, 9(5), 5-9.

Kugel, P. (1993). How professors develop as teachers. Studies in Higher Education. 18 (3), 315-328.

Lee, B. A. (1991). Campus Leaders and Campus Senates. New directions for Higher Education, 13(3), 41-61.

Middlehurst, R. (2004). Changing internal governance: a discussion of leadership roles and management structures in UK universities. Higher Education Quarterly, 58 (4), 258-279.

Miller, M. (1999). Conceptualizing Faculty Involvement in Governance, in $\mathrm{M}$. T. Miller (ed) Responsive Academic Decision-Making: Involving Faculty in Higher Education Governance. Stillwater: New Forums Press.

Mintzberg, H (1984). La estructuración de las organizaciones. Barcelona: Ariel.

Moore, M. (1975). An Experiment in Governance: The Ohio Faculty Senate. Journal of Higher Education, 46 (4), 365-379.

Mora, J-G. (2000). El gobierno de las universidades: entre la autonomía y la eficacia, en International Seminar on
University Governance and Management. Barcelona: EAIR.

Oja, S. (1989). Teachers: ages and stages of adult development, en Holly, M.L \& McLaughlin, C.S. (Eds.) Perspectives on Teacher Profession Development. London: Falmer Press.

Oxford University (2006). White Paper on University Governance. Oxford University Gazette, 36 (Supp. 5).

Pfnister, A. (1970). The Role of Faculty in University Governance. Journal of Higher Education, 41 (May/June), 430449.

Ramsden, P. (1998). Learning to lead in higher education. London: Routledge.

Ribbins, P. (2007). Middle leadership in schools in the UK: improving design a subject leader's history. 10 (1), 13-30.

Robertson, D.L. (1999). “Professors' perspectives on their teaching: a new construct and development model. Innovative in Higher Education, 23 (4), 271-294,

Robbins, S. (1987). Comportamiento organizacional. Conceptos, controversias y aplicaciones. Madrid: Prentice Hall.

Samper,L. (1992). Cultura Professional y ciclo vital: continuidad y cambio en la socialización ocupacional de los docentes. Revista de Sociología, 39, 1121.

Seminar Report (2008). Future business models for universities in the UK: issues and challenges. Universities UK 1-2 May.

Smith, D. (2008). Academics or executives? Continuity and change in the roles of pro-vice-chancellors. Higher Education Quarterly. 62 (4), 340-357

Sporn, B. (2001). Building adaptative universities: emerging organizational 
forms based on experiences of European and US universities. Tertiary Education and Management, 7, 121-134

Sporn, B. (2003). Management in Higher education: current trends and future perspectives in European colleges and universities. Higher education Research and practice, 97-107.

Tesch, R. (1990). Qualitative Research. NY: Falmer.

Trackman, L. (2008). Modeling University Governance. Higher Education Quarterly, 62, 63-83.

Trow, M. (1989). Continuidad y cambio en la educación superior norteamericana, en Burgen, A. (Ed.). Metas y proyectos de la educación superior. Una perspectiva internacional. Madrid: Fundación Universidad-Empresa.
Turnbull, S. \& Edwards, G. (2005). Leadership development for organizational change in a New UK University. Paper presented at the Academy of $\mathrm{Hu}$ man Resource Development International Conference (AHRD) (Estes Park, CO, Feb 24-27, 2005), 109-116

Williams, D. (1987). One Faculty's Perception of Its Governance Role. Journal of Higher Education, 58 (6), 629. 657.

Zabalza, M.A (2002). La enseñanza universitaria. El escenario y sus protagonistas. Madrid: Narcea.

Zepke, N. (2007). Leadership, power and activity systems in a HEW context: will distributive leadership serve in an accountability driven world? International Journal Leadership in Education, 10 (3), 301-314. 


\section{PALABRAS CLAVE}

Universidad, Carrera académica, Profesorado gestor.

\section{KEY WORDS}

University, Development career, Academic-manager

\section{PERFIL ACADÉMICO DE LOS AUTORES}

Diego Castro es diplomado en Educación Social y licenciado en Pedagogía. Master en Dirección de Recursos Humanos y Doctor en Ciencias de la Educación. Es profesor del Departamento de Pedagogía Aplicada de la Universidad Autònoma de Barcelona. Está especializado en el ámbito de la gestión de centros siendo una de sus líneas de trabajo principal la gestión y el gobierno universitario y sus procesos de innovación y mejora.

Marina Tomás, maestra, licenciada en Pedagogía y Doctora en ciencias de la Educación por la Universidad de Barcelona. Ha escrito numerosos libros y artículos en revistas españolas e internacionales. Profesora Titular de la Universidad Autónoma de Barcelona. Dirige desde 1999 el equipo CCUC (Cambio de Cultura en la Universidad Contemporánea) que gestiona diferentes investigaciones regionales, nacionales y europeas sobre la Universidad.

Dirección de los Autores: Diego Castro y Marina Tomás

Departamento de Pedagogía Aplicada, G-6, 244 y 268.

Facultad de CC. De la Educación.

Campus de Bellaterra.

(08193) Cerdanyola del Valles

E-mail: diego.castro@uab.cat marina.tomas@uab.cat

Fecha Recepción del artículo: 18. Septiembre. 2009

Fecha Revisión del artículo: 26. Febrero. 2010

Fecha Aceptación del artículo: 24. Marzo. 2010 